\title{
Artelogie
}

Recherche sur les arts, le patrimoine et la littérature de I'Amérique latine

16 | 2021

Fotografía y migraciones, siglos XIX-XXI.

\section{Trois frontières. Le réel, le familial et la peau dans les mémoires photographiques de la post-dictature argentine}

Natalia Fortuny

\section{(2) OpenEdition}

Édition électronique

URL : https://journals.openedition.org/artelogie/9305

DOI : 10.4000/artelogie.9305

ISSN : 2115-6395

Éditeur

Association ESCAL

Référence électronique

Natalia Fortuny, «Trois frontières. Le réel, le familial et la peau dans les mémoires photographiques de la post-dictature argentine », Artelogie [En ligne], 16 | 2021, mis en ligne le 27 janvier 2021, consulté le 03 septembre 2021. URL : http://journals.openedition.org/artelogie/9305 ; DOI : https://doi.org/ 10.4000/artelogie.9305

Ce document a été généré automatiquement le 3 septembre 2021.

Association ESCAL 


\title{
Trois frontières. Le réel, le familial et la peau dans les mémoires photographiques de la post- dictature argentine
}

\author{
Natalia Fortuny
}

Texte issu du livre Memorias fotográficas. Imagen y dictadura en la fotografía argentina contemporánea, Buenos Aires, La Luminosa, 2014. Traduit de l'espagnol (Argentine) par Antonia García Castro.

Traduction réalisée grâce au projet UBACYT dirigé par Ana Longoni.

1 Le présent texte porte sur ce qu'on pourrait appeler les mémoires photographiques de la dernière dictature argentine (1976-1983) ${ }^{1}$. Il s'agit d'artefacts visuels réalisés après le retour à la démocratie qui rendent compte de diverses manières d'agencer une histoire traumatique marquée par la disparition forcée de 30.000 personnes pendant cette dictature ${ }^{2}$.

2 L'une des particularités de la disparition forcée, en tant que modalité de la violence d'État, est la difficulté voire l'impossibilité pour l'entourage de la personne disparue de gérer la douleur de l'absence. La disparition compromet le travail de deuil, elle engage une suspension de la mort, une attente, « une pure douleur »3. D'après Ludmila da Silva Catela ${ }^{4}$, la disparition implique : absence d'un corps, absence d'un moment de deuil, absence d'une sépulture. Cette triple absence sera au cœur des luttes pour la justice dans notre pays et on la verra apparaître dans de nombreuses productions artistiques, notamment dans un ensemble d'essais photographiques qui appréhendent de manière troublante le rapport au passé récent.

3 Les mémoires photographiques de la dictature condensent les particularités suivantes et elles sont indissociables : leur qualité de mémoires sociales d'un passé en commun - dans un jeu où interviennent expériences et mémoires individuelles, et 
l'histoire; leur format visuel photographique - avec toutes les potentialités temporelles, esthétiques et politiques propres à ce langage; et leur élaboration artistique - leur production se distingue par la création et la mobilisation de ressources visuelles singulières dans chaque œuvre.

Il s'agit ici de repenser ces photographies de la post-dictature à l'aune d'une interrogation principale : quelles sont les frontières que celles-ci mettent en tension? En postulant qu'il en existe au moins trois. Trois frontières que ces mémoires en images se chargent de traverser, de déplacer et de remettre en question.

\section{La ligne du réel}

5 La première frontière est celle du réel, que l'on peut aborder également comme le passé, l'histoire, la distance, ou le rapport à la vérité. Quelque chose avec laquelle les mémoires photographiques étudiées dialoguent.

Qu'est-ce qui est réel et qu'est-ce qui ne l'est pas quand il s'agit de mémoires ? Peut-on créer - inventer - les traces du passé récent?

7 Il existe peu de marques de la dernière dictature dans le paysage urbain argentin. Parmi celles-ci, une maison de La Plata, attaquée par les forces armées, conserve encore des traces de cet événement. Dans cette maison fut installée pendant un temps dissimulée par un astucieux mécanisme - une imprimerie clandestine destinée à l'édition de la revue "Evita Montonera " 5 . C'est là que vivait la famille MarianiTeruggi. Le 24 novembre 1976 un groupe dit de tâches spéciales a attaqué cette maison pendant une opération qui a duré près de quatre heures. Cinq militants montoneros sont morts à cette occasion et un bébé de trois mois a été enlevé, une petite-fille qu'on cherche depuis. Aujourd'hui, le lieu est une Maison de la mémoire, un Monument historique national et a été déclaré Patrimoine culturel de la province de Buenos Aires.

8 Cette maison apparait dans le travail Calle 30 №1134 (2008) du photographe Hugo Aveta. Au premier regard, on voit ce qui suit : une vieille maison dont les murs et la porte du garage montrent de nombreux impacts de balles. Là où il devait y avoir une fenêtre, il n'y a plus qu'un trou. On songe à l'explosion d'une bombe : des verres brisés sur le sol semblent le confirmer. On voit aussi le cadre vide d'une porte. La scène est plongée dans le noir, seule la maison est éclairée et d'une manière fort étrange : plus proche de l'éclairage cinématographique que de la lumière naturelle, on ne pense pas à l'éclairage urbain ni au flash. De plus, alors que la présence des murs s'impose, la maison est limitée en haut par un ciel noir homogène et en bas par un sol tout aussi sombre, long, unifié. Cela produit une première sensation d'étrangeté : quelque chose ne cadre pas avec la perception automatisée, prête à décoder n'importe quelle photo documentaire d'un site ravagé. À y regarder de plus près, d'autres indices étranges renforcent le soupçon d'anomalie. Par exemple, la maison évoque le passage du temps, les murs sont très abîmés mais pourquoi les verres semblent tout juste tombés sur le sol ? Pourquoi ne voit-on pas les trottoirs ni les maisons voisines ni d'autres parties du quartier? Comment se fait-il que le trottoir reflète de manière impeccable la maison bombardée ? 


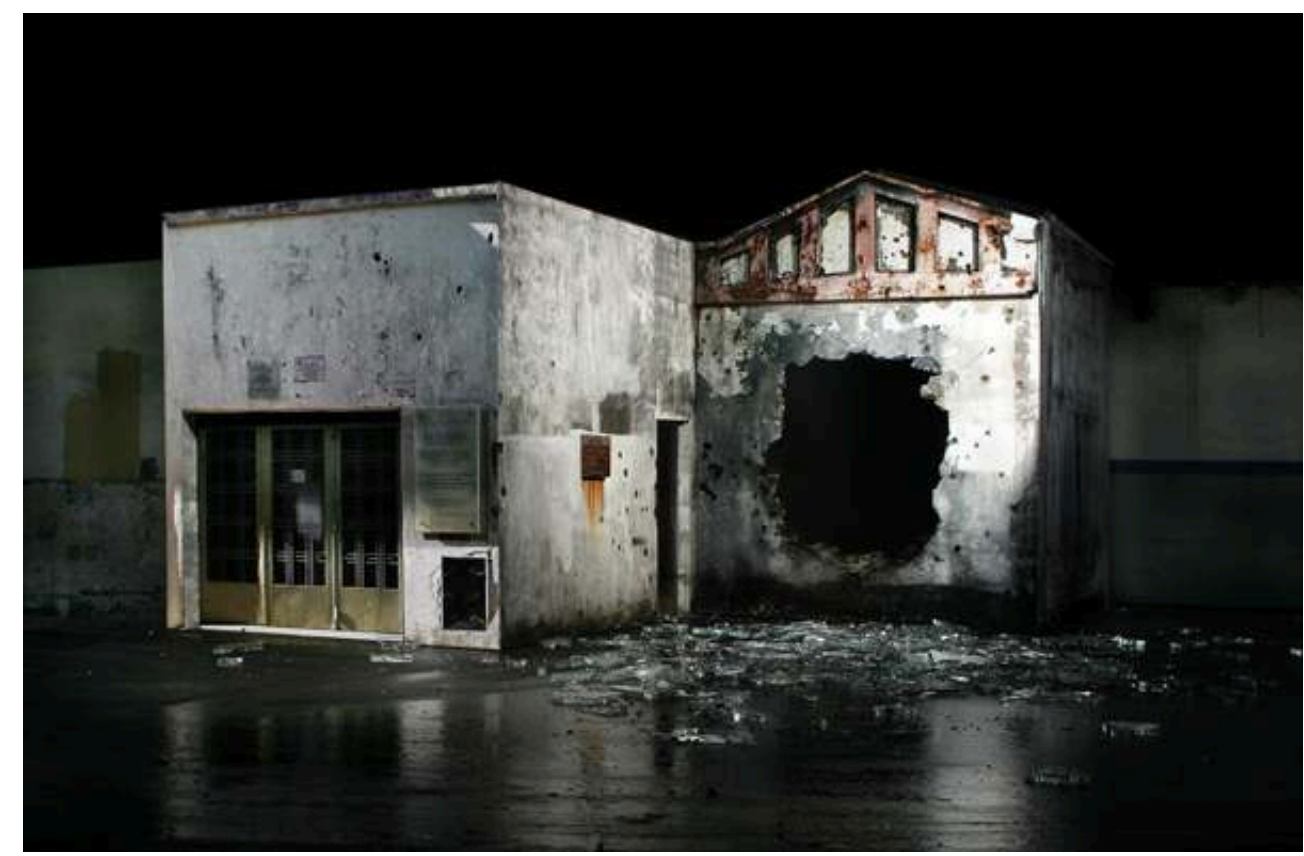

Calle 30 No7134, Hugo Aveta, 2008

9 La réponse est simple : il ne s'agit pas d'une photo de la maison prise in situ mais d'une maquette, de la photo d'une maquette. Le travail d'Aveta ne parle pas seulement du passé mais, pour aborder le passé et surtout la possibilité de construire des mémoires, il parle aussi du dispositif de création du réel - y compris de ce qu'on peut appeler «le réel photographique ». Ce travail problématise la catégorie du réel avec laquelle la photographie a dû combattre depuis des débuts, considérée comme une technique mimétique par excellence. Qu'est-ce qui est réel et qu'est-ce qui ne l'est pas dans cette œuvre? On peut porter sur ce travail deux regards simultanément. D'une part, cette photo montre ce qu'il y a devant la caméra et, en conséquence, quelque chose - la maquette - qui relève du "ça a été » barthésien. D'autre part, cette maquette, cette construction à petite échelle a été photographiée dans un souci de vraisemblance, telle qu'on peut la trouver dans le ferromodélisme. L'effet de sens reste complexe et instable, et fait penser aux procédés de construction du réel.

10 L'artiste parle de cette image en ces termes : «À la croisée des chemins entre réalité et fiction, je travaille sur la perception, les souvenirs et l'histoire sans assener aucune vérité mais sans exclure non plus toute supercherie. (...) Les espaces sont déconstruits et reconstruits (dans les maquettes) sur de nouvelles scènes, en lien avec l'histoire sociale, l'architecture, et ses codes de légitimation et de vigueur. $»^{6}$

11 La photo montre des traces qui sont fictives, des marques inventées de quelque chose qui s'est effectivement produit. Elle présente les traces créées de ce passé traumatique. Comme si certains faits ne pouvaient être évoqués qu'à partir d'une zone ambiguë entre création et fiction. Est-ce que ce n'est pas, en définitive, l'indétermination « maquette/ maison réelle » ce qui provoque l'effet expressif de cette photographie?

12 Cette œuvre vient ici en premier car elle peut être considérée comme ars poetica et synthèse des intentions des mémoires photographiques qui tentent d'évoquer le passé à partir de détours photographiques, de choix esthétiques et de divers dispositifs imaginaires. Ces photographies sont moins des documents que des artefacts sociaux dont la vérité ne réside pas dans l'adéquation à un fait objectivement enregistré mais 
dans leur construction particulière, dans les stratégies et les procédés de production de sens qui les animent. Ce sont des mémoires qui pour évoquer le passé se valent de multiples ressources visuelles propres au fait artistique : le montage, le recyclage, le flou de bougé, la reconstruction, le collage, les intertextualités, entre autres.

13 Les photos conjuguent leur double possibilité d'être des traces du monde et de déployer d'autres mondes métaphoriques et construits. Ainsi, la duplicité propre à l'outil photographique - indice et métaphore - explose dans ces artefacts mémoriels et dans leurs procédés de construction du passé. Selon Jorge Ribalta ${ }^{7}$ l'autonomie problématique de la photographie - à mi-chemin entre l'autonomie des beaux-arts et de l'instrumentalité archivistique - en fait un outil particulièrement adapté pour proposer des possibilités d'articulation entre art et politique. Dans ces séries, le caractère politique des artefacts photographiques trouve ses possibilités les plus variées.

\section{Mémoires familiales}

14 La deuxième frontière renvoie au fait de traverser le seuil de l'intime, l'univers familial et privé. Ici entre en scène un type singulier d'archives domestiques classées par plusieurs mains sur de longues années : les photos de famille. Plusieurs artistes - ayant des liens de parenté direct avec des personnes disparues - ont recours aux albums photos qu'ils utilisent comme déclencheur de nouvelles images. À rebours de leur fonction première, ils les réutilisent pour les mener vers la sphère publique et souligner une absence.

Une des premières séries photographiques qui a eu recours à des photos de famille pour évoquer l'absence d'une personne disparue est Arqueología de la ausencia (1999) de Lucila Quieto. Ces photos reconstruites ont été pensées à partir d'une double absence: la disparition de son père alors que sa mère était enceinte d'elle et l'absence d'une photo avec son père dans l'album de famille.

16 «Ce que je dois faire », s'est-elle dit, «c'est entrer dans l'image, construire l'image que j'ai toujours cherchée $»^{8}$. En 1999, elle a scanné les images qu'elle avait de son père, elle les a projetées sur le mur, puis elle s'est mise au milieu pour prendre une nouvelle photo, une image double et impossible, capable de les contenir pour la première fois tous les deux. Après avoir vu le résultat et la réaction émue de certains de ses camarades, elle a lancé une invitation à d'autres fils et filles de disparus pour qu'ils puissent avoir aussi leur " photo rêvée ", selon l'annonce qu'elle a fait circuler dans le groupe H.I.J.O.S. ${ }^{9}$ où elle militait. Et c'est ainsi que le jeu avec les photos a pris progressivement une dimension collective. 


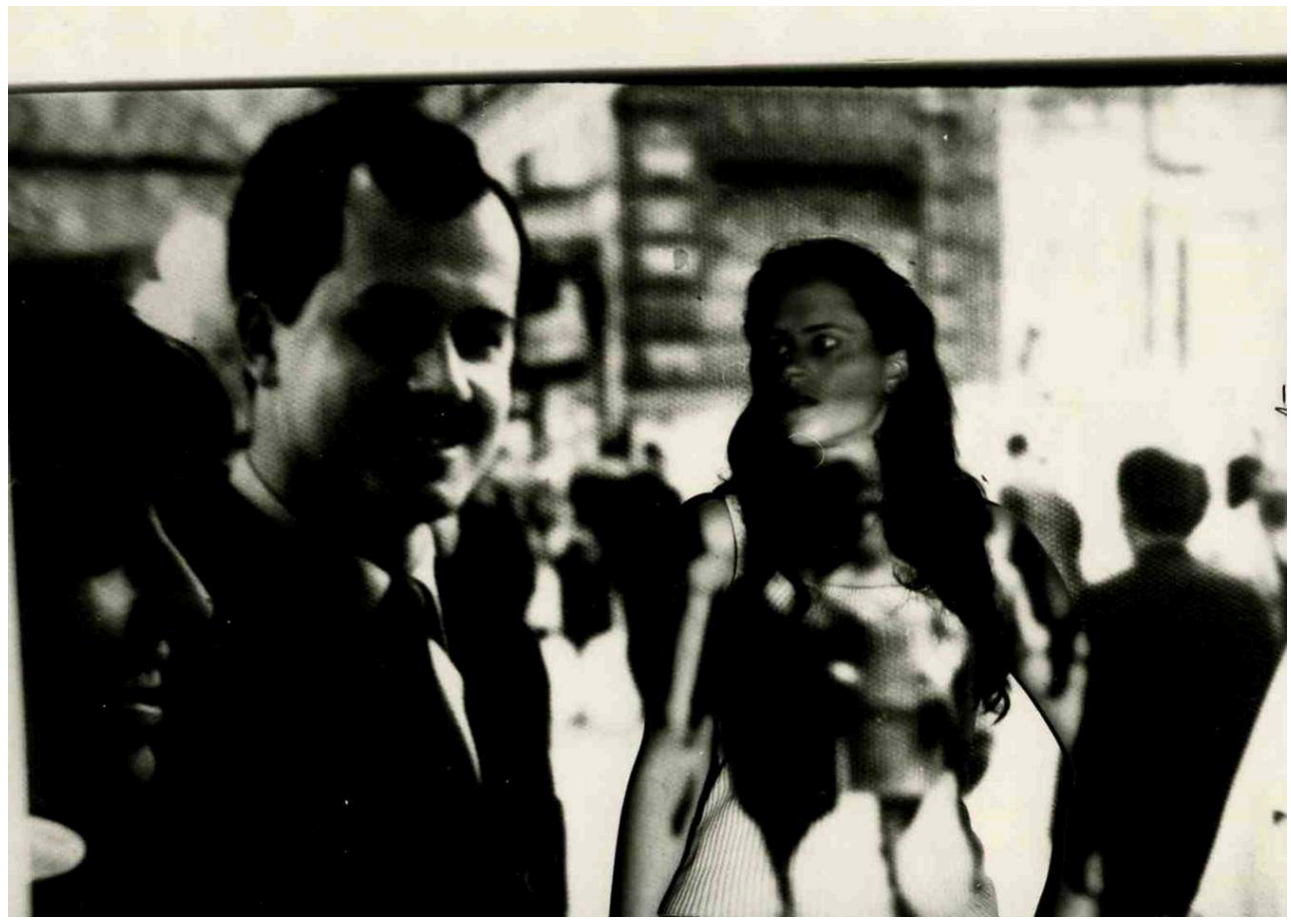



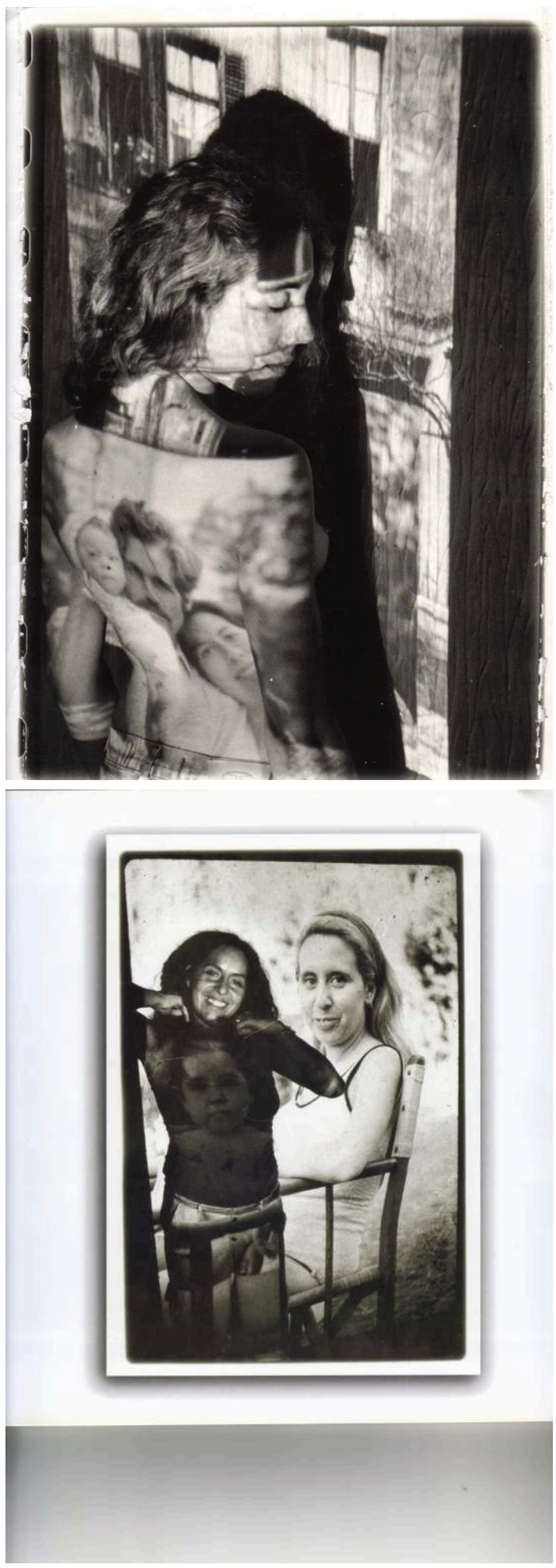

Arqueología de la ausencia, Lucila Quieto, 1999

17 Le monde projeté dans ces photos est perçu tel quel. C'est le summum de l'artifice de la série : montrer les coutures de cette rencontre impossible entre des pères, des mères et des fils et filles. Les différences entre les deux matérialités - entre le faisceau de lumière de la projection et le corps du fils ou de la fille pris dans tout son volume - font que ces rôles se maintiennent exception faite d'un déplacement : le montage n'est pas 
caché mais exposé pour mettre en évidence le rendez-vous manqué. Ainsi, par exemple, les vieilles photos projetées soulignent l'existence de la photo en tant qu'objet préservé et marqué par ces fils et filles qui depuis des années, regardent et touchent la seule photo de leur père ou leur mère disparu(e).

Les photos reconstruisent la scène familiale impossible - la rencontre qui n'a pas pu avoir lieu avec le père et/ou la mère - et, en même temps, elles constituent un témoignage collectif en présentant des photos de -et pour- d'autres fils et filles de disparus. Ainsi, de même qu'Antigone prend une dimension politique au moment où elle revendique des liens de sang, ces photos reconstruites de fils et filles traversent la scène domestique de chaque album familial. Ce qui s'affirme alors c'est le fait qu'il ne s'agit pas seulement de mémoires privées mais profondément politiques et sociales, marquées par leur temps historique.

Jouant avec l'histoire et la politique, les travaux de Lucila Quieto habitent au royaume des travaux pratiques, de la photographie artisanale et du montage. Elle coupe et monte des photos en sachant, qu'une fois remontées, les jointures se verront sur la peau des fils et filles comme des cicatrices.

20 La série El viaje de papá (2005) de Camilo del Cerro constitue, selon les mots de l'artiste, un «essai photographique réalisé par photomontage à partir de photos de voyage, prises dans les années 1960 par Hernán Pérez del Cerro, et d'autoportraits de moimême, son fils $»^{10}$. Chaque photo comprend par ailleurs une légende écrite à la main qui fait partie de l'œuvre : ce sont des extraits d'une lettre que sa tante Magdalena a écrit à son père un mois après son assassinat survenu le 9 juin 1977.

Les vingt photos qui composent cette série montrent des intérieurs d'hôtels ou des pensions, des hommes avec des turbans, des orientaux, des pêcheurs, une mosquée, des enfants qui sortent nus de la mer. Dans presque toutes ces photos, on voit un homme jeune et mince qui porte la barbe : c'est le père disparu de Camilo qui prend des photos de son voyage autour du monde. Dans dix photos, par photomontage, Camilo entremêle sa propre image aux photos où apparaît son père. Le fils se glisse tantôt dessous, tantôt derrière, et devient fantôme, portrait sur le mur, invité, acteur, spectateur. L'apparition en couleur du fils dans les photos en noir et blanc du père est à la fois étrange et naturelle, tout comme la ressemblance entre ces hommes qui ont à peu près le même âge. 


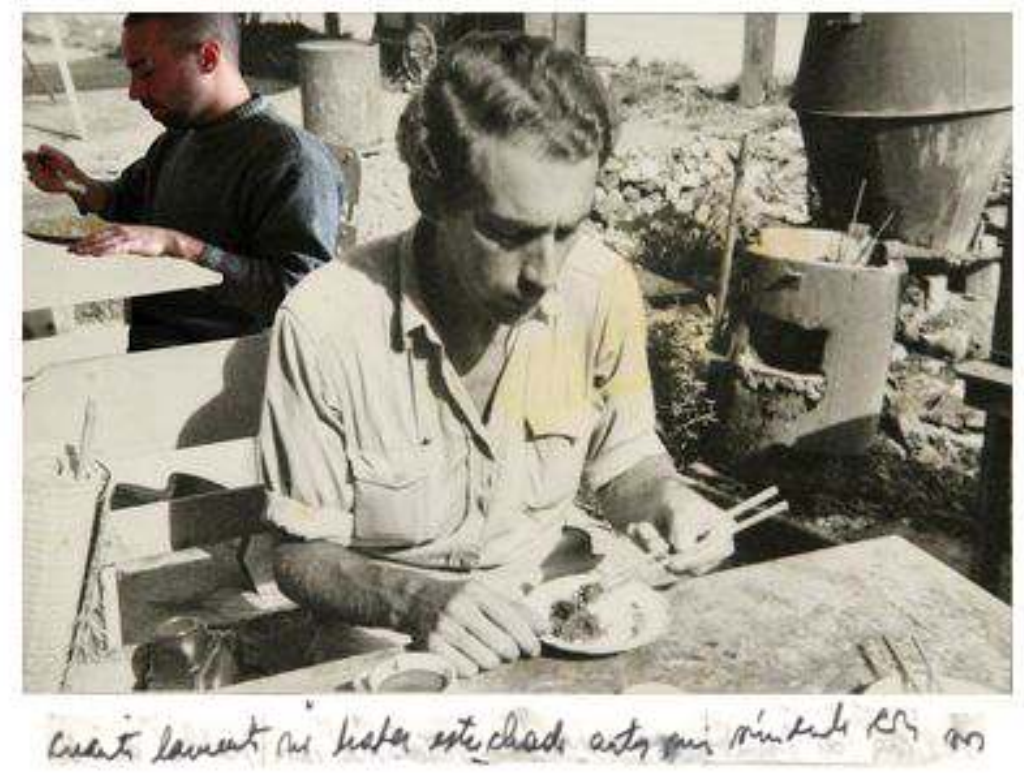

El viaje de papá, Camilo del Cerro, 2005 familial - certaines sont antérieures à la disparition de son père - et les mêle à de nouvelles photos et des poèmes dont elle est l'auteur. Il y a des photos d'arbres, des photos de famille, un lac, des montagnes, deux enfants dont l'image est floue, une Renault $4 \mathrm{R}$ et son chauffeur, une grande maison, une petite-fille dans un bateau de la main de son père. Il y a aussi de brefs textes poétiques placés entre les images, qui offrent des souvenirs et des anecdotes vagues où coexistent des voix de petite-fille et de femme, qui se mêlent aux bruits de l'eau et du bois. Les paysages photographiques/ 
poétiques qui conforment cette œuvre provoquent malaise et empathie. Dès les premières pages, Gaona introduit le lecteur/regardeur aux images qu'il va trouver au moyen d'un bref texte poétique qui fait office de préface esquive. Le texte explicite sans expliquer que lors de ces vacances dans le sud ils ont pris une photo particulière, une image-amulette ${ }^{11}$. Le seul portrait de Gaona avec son père disparu peu après ces vacances sera le nœud et le soutien du livre. Cette image du bateau est entourée d'autres images. Celles «d'avant» ont été prises à l'occasion d'autres vacances de la famille dans leur maison de Bariloche, avant et après la disparition du père. Coexistent avec ces photos issues de l'album, d'autres photos, mélangées. Ce sont les photos que Gaona, devenue adulte et photographe, prend des années plus tard lorsqu'elle retourne à la maison où elle passait ses vacances en famille. Rien - hormis les indices chromatiques, les poses et les vêtements - n'explicite dans le livre quelles sont les vieilles photos et les nouvelles. 

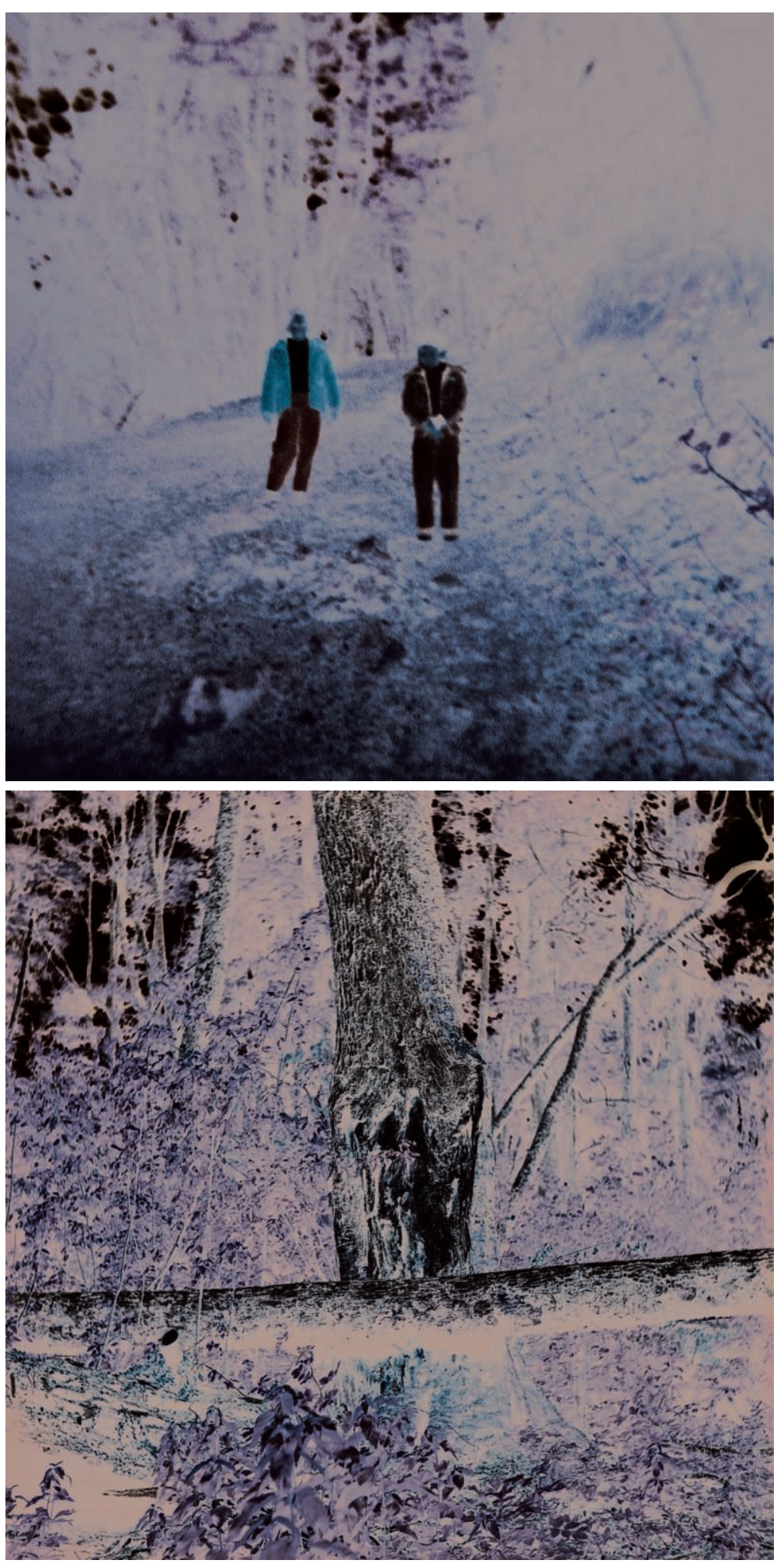

Pozo de aire, Guadalupe Gaona, 2009

En tant que fille de disparu - de même que d'autres artistes qui ont aussi cette filiation - Gaona part d'un manque et d'un creux : l'absence de son père, renforcée par l'absence de photos avec lui. Ainsi, elle s'en va chercher avec son appareil ce qu'elle n'a pas : des photos. Au cours de cette sortie, appareil photo et album à la main, elle va creuser pour tâcher de voir où était ce bonheur, le rayon de lumière de ce passé qui s'échappe pour 
toujours. Gaona prend des photos pour trouver et reconstruire quelque chose de cette image du bateau, quelque chose du moment heureux dans ce resplendissement fuyant du passé. Pour voir si quelque part dans ce paysage apparaissent les marques, les traces de ces vacances et des photos. En essayant, à la manière de Walter Benjamin, d'attraper le passé non « comme il s'est réellement passé » mais comme un éclair juste avant qu'il ne s'échappe.

Dans Recuerdos inventados (2003) Gabriela Bettini présente - et se présente avec - son grand-père et son oncle disparus. Ex militante du siège madrilène de H.I.J.O.S., elle est petite-fille, nièce et arrière-petite-fille de disparus. A cette triple identification par le lien familial s'ajoute le fait qu'elle et ses parents s'exilent en Espagne lorsque ces disparitions se produisent.

Dans cette série photographique, Bettini introduit un jeu ludique et théâtral en présentant l'oncle et le grand-père - leurs portraits photographiques - en dialogue avec le corps de l'artiste. 


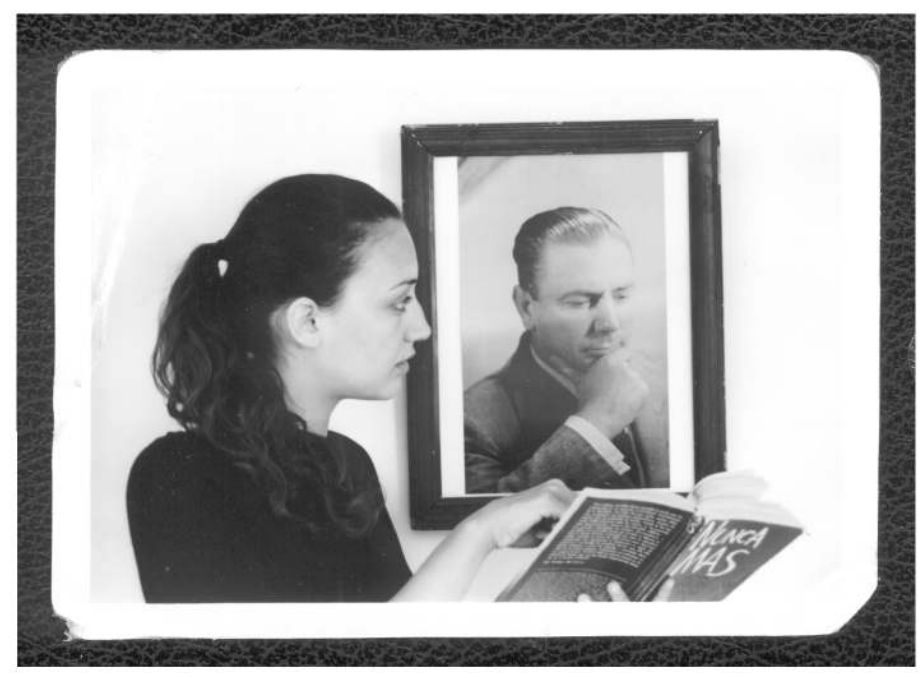

Conversación con Antonio

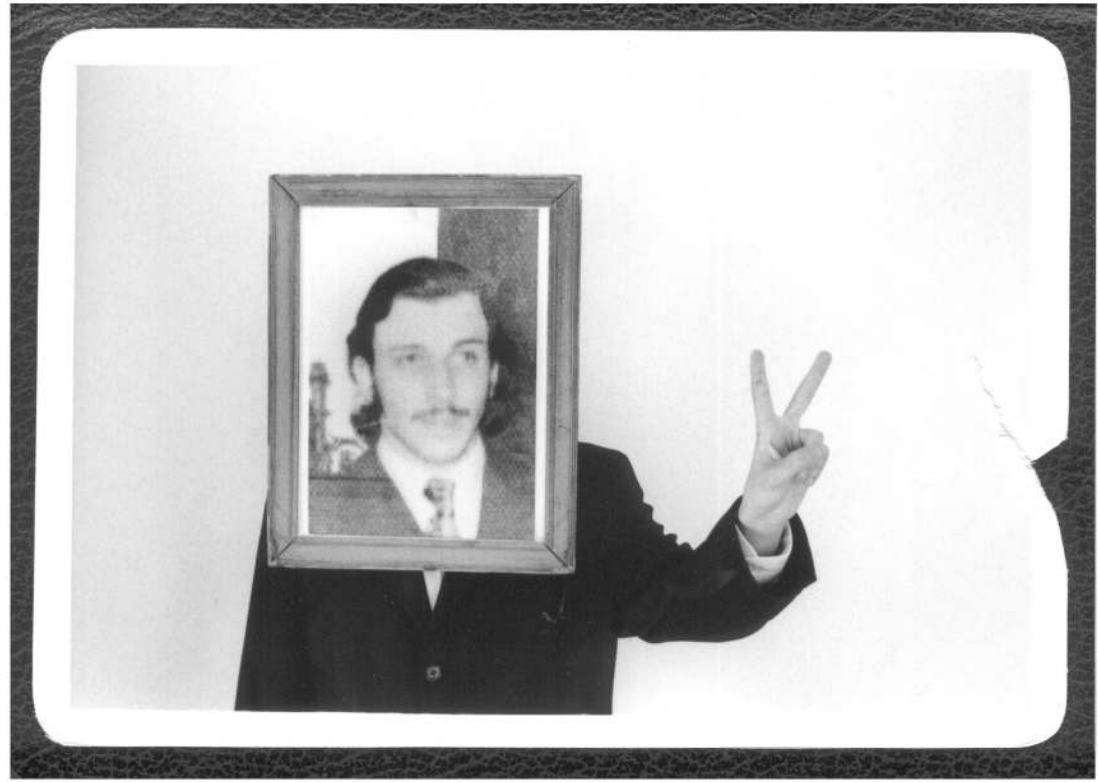

Mi tío Marcelo

Recuerdos inventados, Gabriela Bettini, 2003

L'évocation du corps absent passe ici par la reconstruction à partir d'un autre corps : un corps réel avec une photo qui remplace sa tête. En absence du corps du disparu, il faut lui en trouver un, doter d'un corps quelconque cette tête/photographie, le réanimer. Bettini choisit les photos des disparus et les place de telle manière que les gestes de ceux dont elle fait le portrait dialoguent avec elle : ils lui sourient, ils ont l'air de lire le Nunca más ${ }^{12}$ grâce à elle, ils regardent là où elle leur indique ou bien font le salut péroniste. La mise en scène travaille de manière subtile avec l'humour noir en 
concédant un nouveau corps au disparu. Le portrait de l'absent est poussé à un point limite qui met mal à l'aise ou fait rire.

L'arrière-fond de ces fausses photos de faux moments vécus par le grand-père, l'oncle et la petite-fille/nièce révèle la vérité de l'impossible. Ces photos existent en tant que preuves de leur impossibilité. L'artifice évident du mécanisme le confirme. Ces portraits et ces autoportraits sont vrais en tant que fictions, en tant qu'autofictions qui visent l'absence et ce qui n'a pas pu être vécu du fait de l'irruption de la violence d'État dans la vie familiale. Ce sont des images de situations hypothétiques - une discussion, des vacances - conçues, selon l'artiste, pour «réparer ». Une fois encore, le pouvoir reconstructeur et réparateur de la photographie vient suppléer l'absence et son corrélat : le manque de photos et autres souvenirs. Selon ses mots, Bettini a dû inventer «ce qu'aurait pu être la relation avec ces personnes si je les avais connues. C'est lié à l'idée de compléter des souvenirs qui pour moi sont incomplets $»^{13}$. Et c'est dans cette optique qu'elle a recours à des souvenirs photographiques fictifs - bien que tout souvenir soit une fiction.

Ces photographes qui sont aussi des fils et filles de disparus ont recours à leur propre album de famille - un album significativement incomplet - pour produire de nouvelles photos, plus complexes dans leur statut privé-public et à la croisée des rituels intimes et des luttes collectives. Ces œuvres exposent, outre la construction propre à chaque image, une reconstruction particulière et un jeu temporel avec cet instant attrapé/enfui pour toujours.

\section{Écrit sur la peau}

La troisième frontière est une frontière de vie. C'est la peau en tant qu'organe le plus vaste du corps humain, intérieur/extérieur, touchant/touché, qui scelle et sauvegarde chaque intériorité tout en nous connectant les uns avec les autres. La peau est paysage unique et archive des itinéraires personnels. Superficie de la sculpture du monde en nous, palimpseste qui mue en permanence, la peau dans sa fragilité nous enlace avec l'en dehors.

32 Les peaux se montrent comme des superficies privilégiées de l'inscription du passé dans quelques-unes de ces mémoires photographiques. C'est le cas, par exemple, de la série déjà citée de Lucila Quieto, et de deux autres séries que nous allons examiner.

Visuellement liée à la série de Quieto, dans El rescate (2007) Verónica Maggi présente un ensemble de photos en couleur de sa mère disparue projetées sur son corps, devenue scène susceptible d'abriter les trésors du passé. Maggi utilise aussi les photos de famille pour réaliser des artefacts esthétiques/temporels d'un autre ordre et supporte l'œuvre sur certaines parties de son corps nu. 


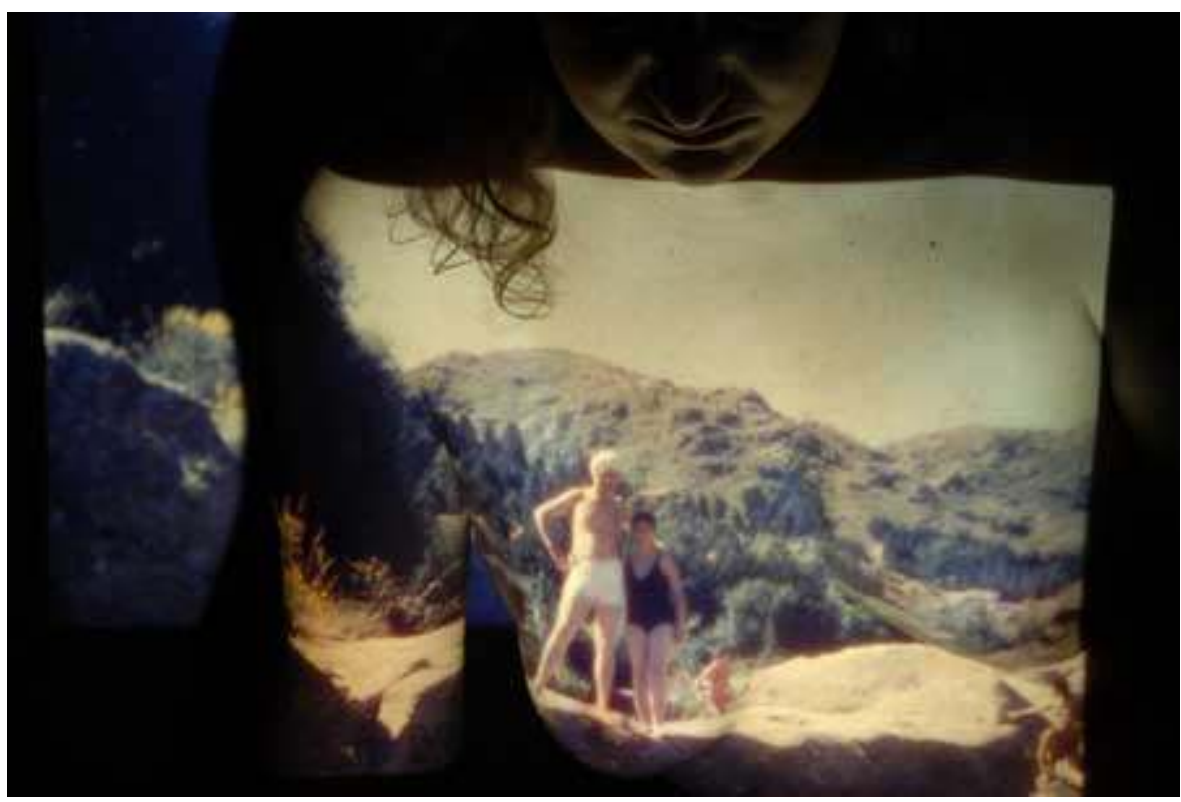

El rescate, Verónica Maggi, 2007 coction sur son propre corps de l'image de la mère, est sans doute aussi une quête qui permet de réunir le passé de la fille - vivante - et le futur de la mère - assassinée. Tout comme dans les photos de Quieto, Gaona et Del Cerro, plusieurs temps se rejoignent de manière anachronique et s'interconnectent dans une même image grâce au montage.

peau des fils et filles apparaît aussi dans un diptyque de l'exposition et du livre Imágenes en la memoria (2011) de Gerardo Dell'Oro, frère de Patricia, jeune disparue. Dell'Oro, fils de photographe, raconte l'histoire de vie de sa sœur, à partir de photos prises par leur père - son enfance, son adolescence, son mariage - et des photos qu'il a lui-même pris de sa nièce, la fille de Patricia séparée de sa mère au moment de son arrestation alors qu'elle n'avait que quelques jours ${ }^{14}$. 

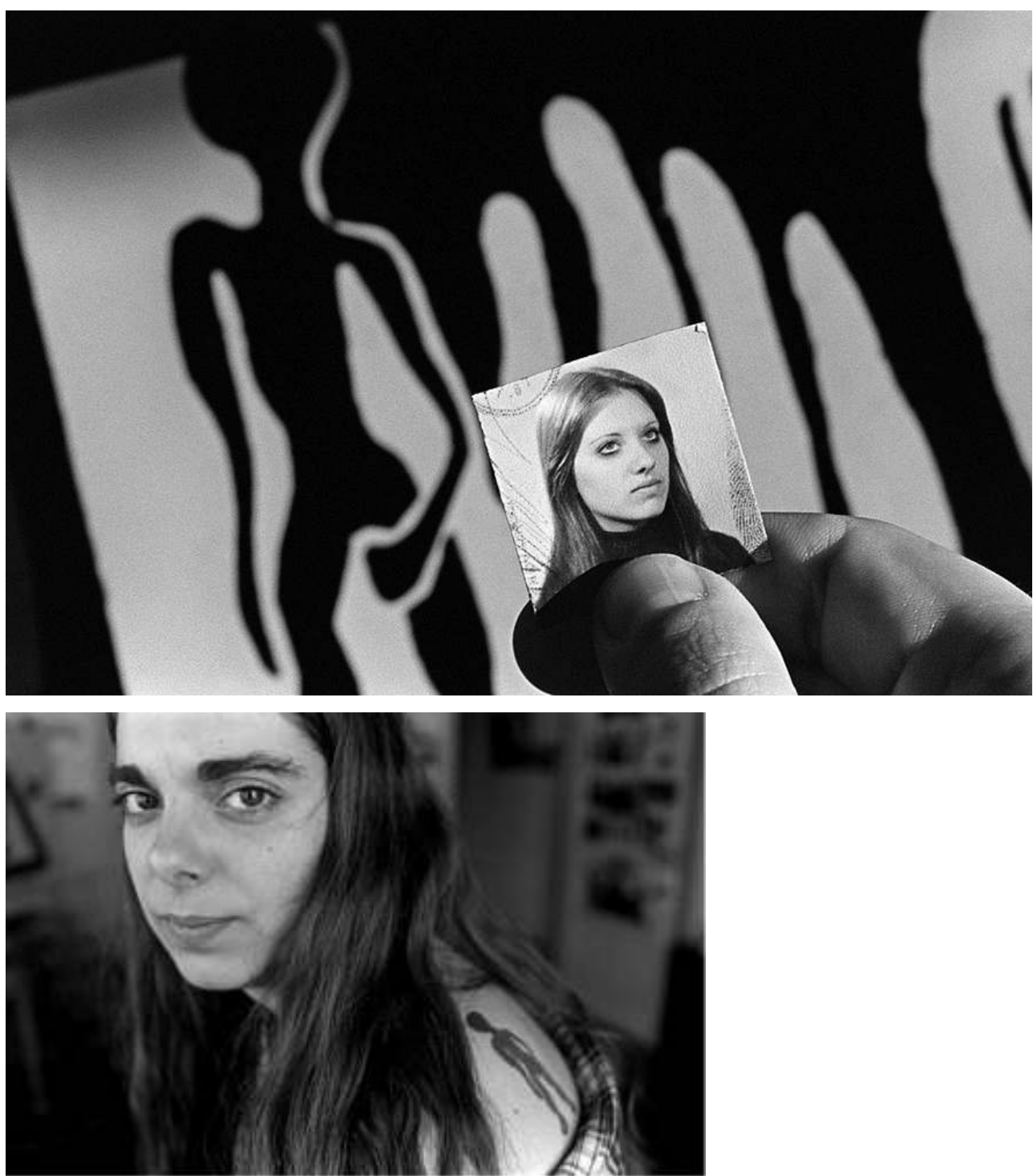

Imágenes en la memoria, Gerardo Dell'Oro, 2011

38 Ce qui frappe dans cette série, c'est la grande ressemblance physique de la mère et la fille: le visage, les cheveux, la coiffure, les yeux. Tout cela accentue la continuité narrative entre la mère et la fille que les images proposent, un peu comme s'il s'agissait d'une quête des traces de la mère-sœur dans la fille-nièce. À deux reprises, la photo de Patricia apparaît dans l'image: entourée de bougies, et dans une photo d'identité soutenue par une main alors qu'en arrière-plan on voit le dessin d'une silhouette. Cette silhouette fait partie d'un tableau peint par la mère, et comme on peut l'apprécier dans une autre image, la fille le porte tatoué sur l'épaule.

39 Dans sa série, Dell'Oro reconstruit la biographie de sa sœur à partir du regard de son père, et la biographie de sa nièce, fille de disparus, à travers son propre regard. En même temps, il rend compte de l'horreur en explorant visuellement des scènes sinistres ainsi que le témoignage des tortures infligées à sa sœur et à son beau-frère, et de leur assassinat.

40 Bien que tout album soit probablement fait pour être commenté et recomposé selon le récit que l'on fait à son propos, sa vocation n'est pas, dans la plupart des cas, d'être exposé au public. Et c'est précisément ce mouvement que tentent ces artistes dans leurs 
travaux. Les peaux nues sont le support matériel des images du passé : c'est la trace des parents (la «trace familiale ») sur les corps de fils et filles qui laisse une marque réelle, une trace de lumière indélébile.

Exercices photographiques

41 Avant de conclure, je voudrais évoquer une œuvre de l'artiste colombien Óscar Muñoz. La vidéo-installation Proyecto para un memorial enregistre l'exécution d'une tâche inutile : Muñoz en train de dessiner sur le sol chaud de Cali des portraits avec de l'eau ; des portraits tirés de la rubrique nécrologique d'un journal. Lorsque vient le moment de compléter le dessin, les premiers traits s'estompent, s'évaporent, il faut recommencer. Cette action qui consiste à dessiner avec de l'eau sur une pierre chauffée au soleil les visages des morts qui disparaissent sans cesse et qui est toujours recommencée - en boucle, comme une tâche qu'on ne peut ni interrompre ni achever - sert à souligner le persistant travail de la mémoire depuis l'instant présent et avec l'image. Un travail répété - peut-être vain d'un certain point de vue - qui ne cesse de recommencer, et qui engage le spectateur dans un exercice de remémoration éphémère mais obstiné.

Il existe un grand nombre de travaux argentins consacrés au passé récent dictatorial qui sont de véritables efforts de mémoire et qui interpellent - à la manière de Muñoz le spectateur dans des lieux non évidents et non cristallisés. Les appréhender en termes de mémoires photographiques s'avère fructueux pour ces productions qui sont à la fois des mémoires sociales d'un passé en commun, des artefacts photographiques - avec leurs particularités temporelles, esthétiques et politiques - et des élaborations artistiques créatives qui mettent en branle des ressources visuelles singulières.

Parce que l'image peut aussi avoir pour enjeu de monter, de démonter et de remonter. De jouer avec les frontières. En comprenant toujours la photographie comme une construction, comme la création d'un dispositif qui la rapproche de l'artifice (et qui met en tension son caractère indiciel) pour conserver néanmoins, dans tous les cas, cette étrange et riche racine qu'une photo entretient avec les choses, le monde et l'histoire.

\section{NOTES}

1. Traduit de l'espagnol (Argentine) par Antonia García Castro. Traduction réalisée grâce au projet UBACYT dirigé par Ana Longoni.

2. J'ai travaillé ces thèmes dans le livre Memorias fotográficas. Imagen y dictadura en la fotografía argentina contemporánea, Buenos Aires, La Luminosa, 2014. Voir: issuu.com/espacioeclectico/ docs/libro_natalia_fortuny.

3. Schmucler, Héctor, "Ni siquiera un rostro donde la muerte hubiera podido estampar su sello (reflexiones sobre los desaparecidos y la memoria)", Pensamiento de los confines Nro. 3, Buenos Aires, 1996.

4. Da Silva Catela, Ludmila, No habrá flores en la tumba del pasado. La experiencia de reconstrucción del mundo de familiares de desaparecidos, La Plata, Ediciones Al Margen, 2001. 
5. Une partie de cette histoire est racontée dans le premier roman autobiographique de Laura Alcoba qui a vécu, enfant, quelques mois dans cette maison, avant de partir rejoindre sa mère en France. Voir Manèges : petite histoire argentine, Paris, Gallimard, 2007.

6. La citation est extraite du blog d'art Bola de Nieve. Voir http://www.boladenieve.org.ar/artista/ 589/aveta-hugo

7. Ribalta, Jorge, Efecto real. Debates posmodernos sobre fotografía, Barcelona, Gustavo Gili, 2004.

8. Entretien avec Lucila Quieto réalisé par Ana Longoni, Buenos Aires, Inédit, 2009.

9. L'association H.I.J.O.S. (dont les sigles veulent dire : Fils et filles pour l'Identité et la Justice contre l'Oubli et le Silence) s'est formée en 1994 comme un espace de lutte et d'activité militante pour les enfants de disparus de tout le pays. Très vite, ils se sont distinguées par la créativité et le caractère ludique de leurs pratiques de dénonciation.

10. La citation est extraite du site web Indymedia Argentina. Voir http://argentina.indymedia.org/ news/2005/12/354887.php

11. Gaona, Guadalupe, Pozo de Aire, Bahía Blanca, Vox Senda, 2009.

12. Le Nunca más (Jamais plus) est le rapport final de la Commission nationale sur la disparition forcée de personnes (CONADEP), conformée en 1983 par le gouvernement démocratique de Raúl Alfonsín pour enquêter sur les enlèvements, les tortures et la disparition de personnes pendant la dictature militaire.

13. Extrait d'un entretien réalisé par l'auteur de cet article en 2011.

14. Dell'Oro, Gerardo, Imágenes en la memoria, Buenos Aires, La Luminosa, 2011. 